extended by macro-level determinants (wealth of countries, income inequality, health expenditure, welfare state regime-dummies).

Results Boys and girls in the Southern, Eastern and Liberal regimes displayed the highest prevalence rates in multiple complaints in contrast to the social-democratic and conservative regimes. The liberal regime and, thus, countries with high income inequality showed significantly more complaints and a stronger impact of socio-economic determinants on complaints.

Conclusion Reducing health inequalities should continue to be an important public health strategy with emphasis on the young population in all welfare states regimes and particularly in the Liberal welfare states. Thus, welfare state characteristics and the extent of income inequality are considered as relevant factors in tackling socioeconomic inequalities in health of adolescents.

\section{4-1.6 EXPLORING LIFECOURSE RELATIONSHIPS BETWEEN OBESITY AND PSYCHOLOGICAL HEALTH USING THE 1958 BRITISH BIRTH COHORT}

doi:10.1136/jech.2011.142976b.13

N Cable, ${ }^{*}$ E Brunner. University College London, London, UK

This study explored relationships between obesity and emotional adjustment across lifecourse to contribute to an ongoing debate about a causal relationship between obesity and depression.

Using a British birth cohort born in 1958 (Men=1583, Women $=1723$, complete cases), BMI and emotional adjustment collected at age 16,23 , and 45 were used to test possible inter-relationships between obesity and psychological health. Path analysis was used to identify significant pathways between these factors across lifecourse. In addition, we included physical activities at age 16 and 23 in the model to examine the role on BMI and emotional adjustment at age 23 and 45 .

Limited among men, our findings supported the pathway from emotional adjustment to BMI, but not the pathway from BMI to emotional adjustment. Poor emotional adjustment at age 16 was associated with increased BMI at age 23. Surprisingly, poor emotional adjustment at age 23 contributed to a leaner body mass at age 45 . Physical activities at age 16 were associated with increased BMI at age 23. In women, there was nothing to indicate significant relationships between BMI and emotional adjustment at any time points. These findings are independent of emotional adjustment, BMI, physical activities, and socio-economic status at age 11 .

In conclusion, men's psychological health can contribute to their BMI across lifecourse. However, there may be middle aged men who gained weight in their response to poor emotional adjustment in young adulthood as well as those who lost weight. 